\title{
Global convergence in the vulnerability of forests to drought
}

\author{
Brendan Choat $^{1 *}$, Steven Jansen ${ }^{2 *}$, Tim J. Brodribb ${ }^{3}$, Hervé Cochard ${ }^{4,5}$, Sylvain Delzon ${ }^{6}$, Radika Bhaskar ${ }^{7}$, Sandra J. Bucci ${ }^{8}$, \\ Taylor S. Feild ${ }^{9}$, Sean M. Gleason ${ }^{10}$, Uwe G. Hacke ${ }^{11}$, Anna L. Jacobsen ${ }^{12}$, Frederic Lens ${ }^{13}$, Hafiz Maherali ${ }^{14}$, \\ Jordi Martínez-Vilalta ${ }^{15,16}$, Stefan Mayr ${ }^{17}$, Maurizio Mencuccini ${ }^{18,19}$, Patrick J. Mitchell ${ }^{20}$, Andrea Nardini ${ }^{21}$, Jarmila Pittermann ${ }^{22}$, \\ R. Brandon Pratt ${ }^{12}$, John S. Sperry ${ }^{23}$, Mark Westoby ${ }^{10}$, Ian J. Wright ${ }^{10}$ \& Amy E. Zanne $^{24,25}$
}

\begin{abstract}
Shifts in rainfall patterns and increasing temperatures associated with climate change are likely to cause widespread forest decline in regions where droughts are predicted to increase in duration and severity ${ }^{1}$. One primary cause of productivity loss and plant mortality during drought is hydraulic failure ${ }^{2-4}$. Drought stress creates trapped gas emboli in the water transport system, which reduces the ability of plants to supply water to leaves for photosynthetic gas exchange and can ultimately result in desiccation and mortality. At present we lack a clear picture of how thresholds to hydraulic failure vary across a broad range of species and environments, despite many individual experiments. Here we draw together published and unpublished data on the vulnerability of the transport system to drought-induced embolism for a large number of woody species, with a view to examining the likely consequences of climate change for forest biomes. We show that $\mathbf{7 0 \%}$ of 226 forest species from 81 sites worldwide operate with narrow ( $<1$ megapascal) hydraulic safety margins against injurious levels of drought stress and therefore potentially face long-term reductions in productivity and survival if temperature and aridity increase as predicted for many regions across the globe ${ }^{5,6}$. Safety margins are largely independent of mean annual precipitation, showing that there is global convergence in the vulnerability of forests to drought, with all forest biomes equally vulnerable to hydraulic failure regardless of their current rainfall environment. These findings provide insight into why drought-induced forest decline is occurring not only in arid regions but also in wet forests not normally considered at drought risk $^{7,8}$.
\end{abstract}

Sensitivity to drought is fundamentally important in shaping the geographic distribution of individual species as well as communities ${ }^{9,10}$. Drought has underpinned many large-scale forest mortality events over the past century, often in combination with other abiotic and biotic factors ${ }^{1,11}$. Recent evidence suggests rising global temperatures are already amplifying drought-induced forest change and affecting terrestrial net primary productivity ${ }^{12-14}$. The consequences of longer droughts and higher temperatures are potentially dramatic. For example, rapid forest collapse as a result of drought could convert the world's tropical forests from a net carbon sink into a large carbon source during this century ${ }^{8,15}$. Predicting how forests will respond to future climate changes hinges on a quantitative understanding of the physiological mechanisms governing drought stress at the species level. One of the most promising avenues for characterizing the sensitivity of plants to drought stress is by quantifying the strength of the liquid (hydraulic) connection between soil and leaves through the water-transporting xylem tissue.

Cavitation, a phase change from liquid water to vapour, occurs in plants because water transported through the xylem is under negative pressure $^{16}$. The resultant air emboli block xylem conduits and reduce the plant's ability to move water from soil to sites of photosynthesis ${ }^{17}$. Recent evidence indicates that the ability of woody plants to survive and recover from periods of sustained drought is strongly related to their embolism resistance $e^{2,3}$. This property varies widely among species and is largely determined by differences in the structure of the xylem ${ }^{18-20}$. Although xylem structure can acclimate to environmental variation during growth and development, subsequent acclimation of embolism resistance to environmental stress is not possible because xylem conduits are dead at maturity. Embolism resistance therefore represents a critically important trait for defining the limits of drought tolerance across woody species and predicting drought-induced forest decline at regional and global scales.

In drying soil, stomata initially regulate water loss from the leaves to maintain xylem pressure ( $\Psi_{\mathrm{x}}$; measured as water potential below 0 ) within a range that will protect the xylem from extensive embolism ${ }^{17,21}$. As drought continues, stomatal closure slows but does not halt the decline of xylem pressure and hydraulic capacity. If soil water is not replenished before complete hydraulic failure occurs then the plant will desiccate and potentially die. The resistance of a plant to embolism is described by the relationship between xylem pressure and loss of hydraulic conductivity due to conduit occlusion by gas emboli (Supplementary Fig. 1). The $\Psi_{\mathrm{x}}$ at which $50 \%$ loss of conductivity occurs $\left(\Psi_{50}\right)$ is the most commonly used index of embolism resistance. When $\Psi_{\mathrm{x}}$ falls below $\Psi_{50}$ the water transport function of the xylem is markedly impaired and the plant is exposed to considerable risk of accelerated embolism leading to long-term reductions in productivity, tissue damage, and ultimately death ${ }^{2}$. To examine vulnerability of forest biomes to drought-induced hydraulic failure we assembled a database of $\Psi_{50}$ (Supplementary Table 1, including 480 woody species). Site

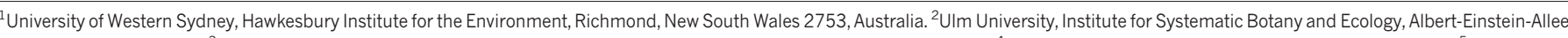
11, 89081 Ulm, Germany. ${ }^{3}$ University of Tasmania, School of Plant Science, Private Bag 55, Hobart, Tasmania 7001, Australia. ${ }^{4}$ INRA, UMR547 PIAF, F-63100 Clermont-Ferrand, France. ${ }^{5}$ Clermont

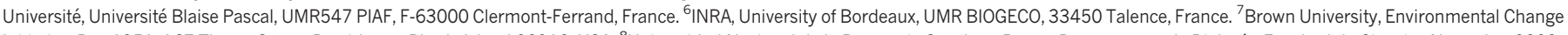

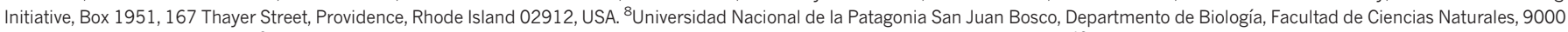

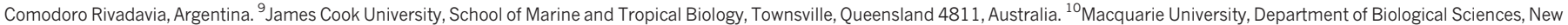

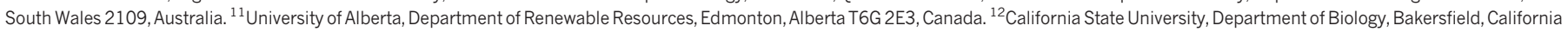

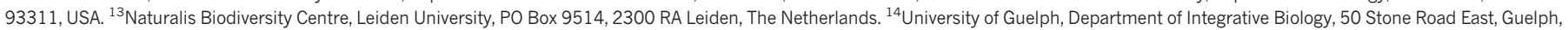
Ontario N1G 2W1, Canada. ${ }^{15}$ CREAF, Cerdanyola del Vallès 08193, Spain. ${ }^{16}$ Universitat Autònoma Barcelona, Cerdanyola del Vallès 08193, Spain. ${ }^{17}$ University Innsbruck, Institut für Botank,

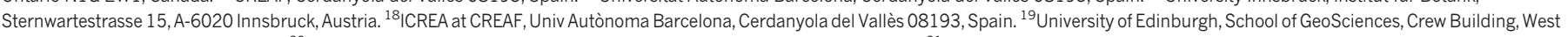

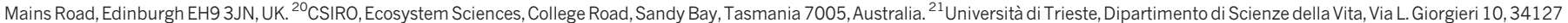

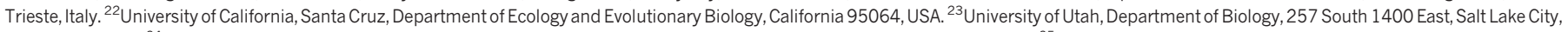

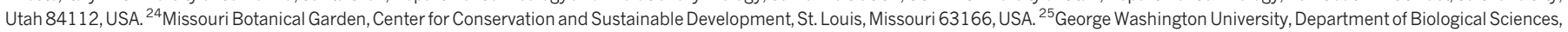
2023 G Street NW, Washington DC 20052, USA.
}

*These authors contributed equally to this work. 


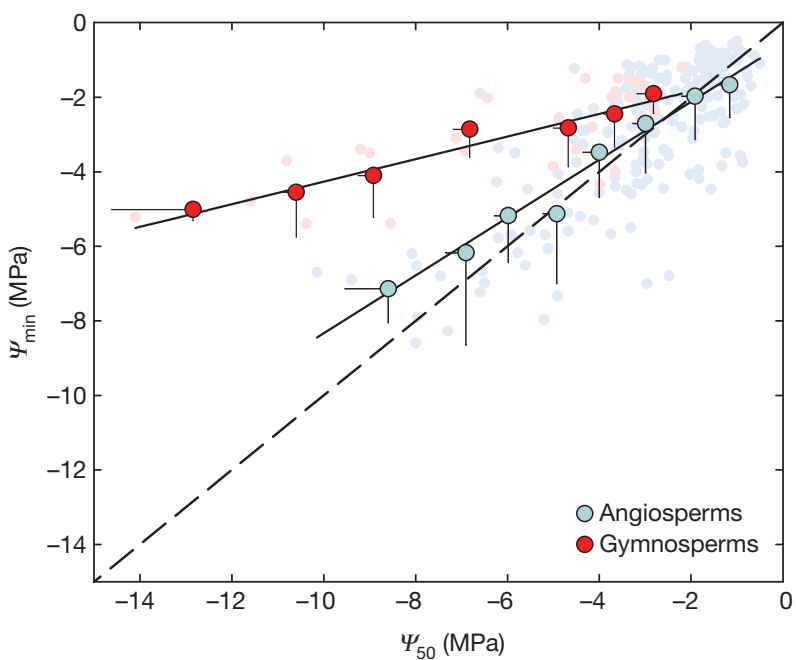

Figure $1 \mid$ Minimum xylem pressure as a function of embolism resistance for 191 angiosperm and $\mathbf{3 2}$ gymnosperm species. The safety margin is the distance between each point and the 1:1 (dashed) line. Data were binned in 1.0$\mathrm{MPa}$ increments for embolism resistance $\left(\Psi_{50}\right)$. Bins were pooled with the next lowest bin if they contained only one sample. Raw data are shown as smaller points behind binned data. Error bars, s.d. Regression lines shown were fitted to raw data (angiosperms, $r^{2}=0.57, P<0.0001$; gymnosperms, $r^{2}=0.59$, $P<0.0001$; data set available in Supplementary Table 1). $\Psi_{\text {min }}$, minimum xylem pressure.

climate varied widely, for example, mean annual precipitation (MAP) ranged from 300 to $4,500 \mathrm{~mm}$, and mean annual temperature from -4 to $27^{\circ} \mathrm{C}$. Data for angiosperms (flowering plants) and gymnosperms (mainly conifers) were analysed separately because of fundamental differences in xylem structure between these two groups.

We observed a significant $(P<0.0001)$ linear relationship between the minimum $\Psi_{\mathrm{x}}$ measured in plants under natural conditions $\left(\Psi_{\min }\right)$ and $\Psi_{50}$ for both angiosperms and gymnosperms, showing that embolism resistance is tightly linked with the level of drought stress experienced by plants across a broad range of environments (Fig. 1). The difference between $\Psi_{\min }$ and $\Psi_{50}$ represents a highly informative 'safety margin' within which the plant operates in a given environment ${ }^{22,23}$. This safety margin quantifies the degree of conservatism in a plant's hydraulic strategy, indicating that plants with low (or even negative) safety margins experience large amounts of embolism and therefore potential risk of hydraulic failure (Supplementary Fig. 1). Measurements of $\Psi_{\min }$ were available for 226 of the 480 species included in our database.

Across all forest biomes, $70 \%$ of all species operated at narrow $(<1$ megapascal $(\mathrm{MPa}))$ safety margins (Fig. 2a), indicating that both arid and mesic biomes are vulnerable to drought-induced decline if extreme drought events become more frequent as predicted under global climate change $e^{5,6}$. Applying more conservative safety margins, for example, the difference between $\Psi_{\text {min }}$ and almost complete xylem failure $\left(\Psi_{88}\right)$, showed a similar convergence of vulnerability across forest biomes (Fig. 2b).

Overall, gymnosperms showed greater hydraulic safety margins than angiosperms, with $42 \%$ of all angiosperms versus only $6 \%$ of all gymnosperms operating at negative safety margins, that is, $\Psi_{\text {min }}$ below $\Psi_{50}$ (Figs 1,2). The seemingly risky embolism-tolerance strategies seen in angiosperms suggest that flowering plants may have a greater capacity to reverse embolism, a process by which gas is dissolved and the conduits are restored to a water-filled and functional status. Although this process is still poorly understood, it is clear that recovery can only occur if periods of drought are followed by sufficient precipitation and a return to favourable water status ${ }^{24,25}$. Therefore, refilling does not represent an effective escape strategy for mitigating the effects of severe and persistent drought.
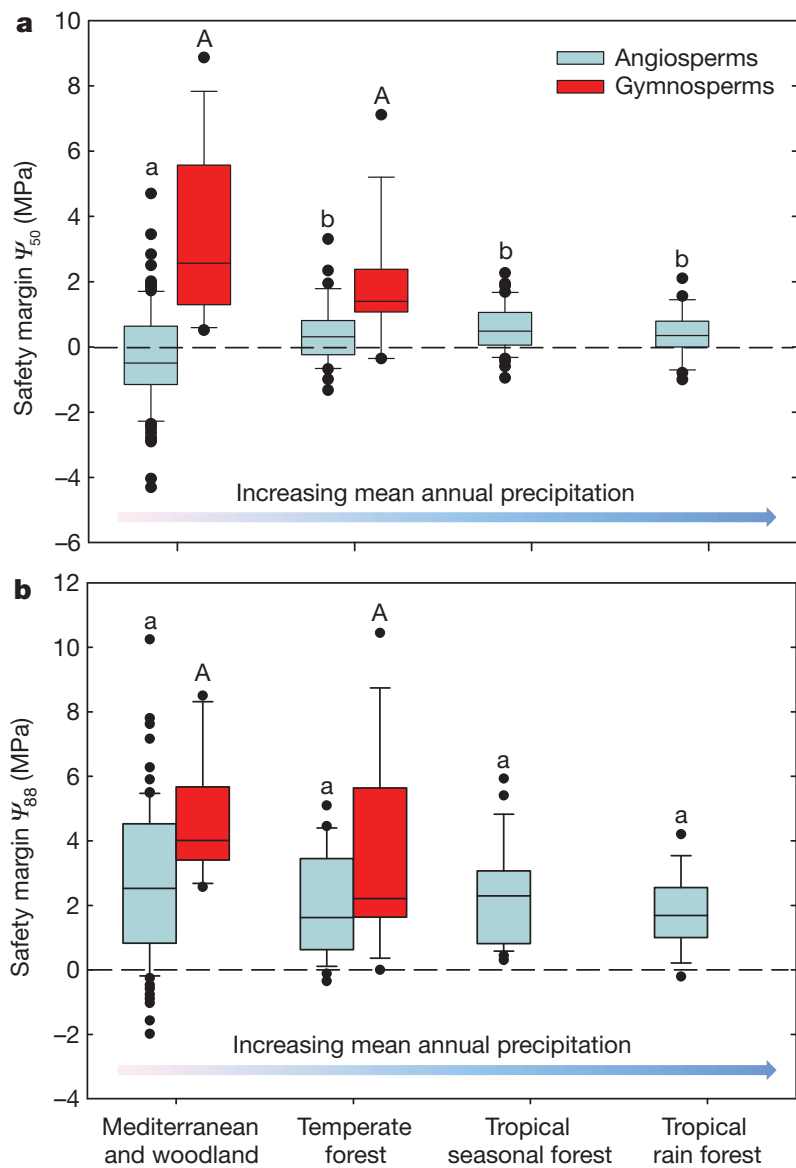

Figure $2 \mid$ Box plot of hydraulic safety margins for angiosperm and gymnosperm species across forest biomes. a, b, The $\Psi_{50}\left(\Psi_{\min }-\Psi_{50}\right)$ safety margin is shown in a $(n=223)$, and the $\Psi_{88}\left(\Psi_{\min }-\Psi_{88}\right)$ safety margin is shown in $\mathbf{b}(n=222)$. Boxes show the median, 25th and 75 th percentiles, error bars show 10th and 90th percentiles, and filled symbols show outliers. Gymnosperm species were not represented in tropical forests. Significant differences $(P<0.05)$ between biome means are indicated by letters above boxes with angiosperms (lowercase $\mathrm{a}, \mathrm{b}$ ) and gymnosperms (uppercase A) considered separately. Data set available in Supplementary Table 1.

Wider safety margins in gymnosperms than angiosperms do not mean that gymnosperms are immune to the threat of hydraulic failure. In fact, Pinaceae species have significantly lower embolism resistance and safety margins than Cupressaceae ${ }^{19,20,26}$, which is reflected in the greater frequency of dieback events involving Pinaceae ${ }^{1,13,26}$. In the severe 2002-2003 drought, for example, Pinus (Pinaceae) species suffered widespread mortality in the south western United States, whereas co-existing Juniperus (Cupressaceae) survived ${ }^{13}$.

In our data set, $\Psi_{50}$ was strongly associated with MAP (Fig. 3) such that both the mean and upper tenth quantile trends showed decreasing resistance to embolism (less negative $\Psi_{50}$ ) with increasing rainfall $(P<0.05)$. Similar relationships were found between $\Psi_{50}$ and climate variables that account for both the variation in potential evapotranspiration (PET) and seasonality of precipitation: aridity index (MAP divided by PET) and mean precipitation of the driest quarter (Supplementary Fig. 2). However, a wide range of hydraulic strategies occur within any given climate region, with the greatest variation in $\Psi_{50}$ occurring at sites with a MAP of between 300 and 1,000 $\mathrm{mm}$. In high MAP sites, represented by tropical rainforests in our data set, variation is compressed to less negative $\Psi_{50}$, suggesting that low embolism resistance is associated with high transport efficiency and low structural 'costs', making this an advantageous strategy in highly productive, wet tropical environments ${ }^{18}$. 


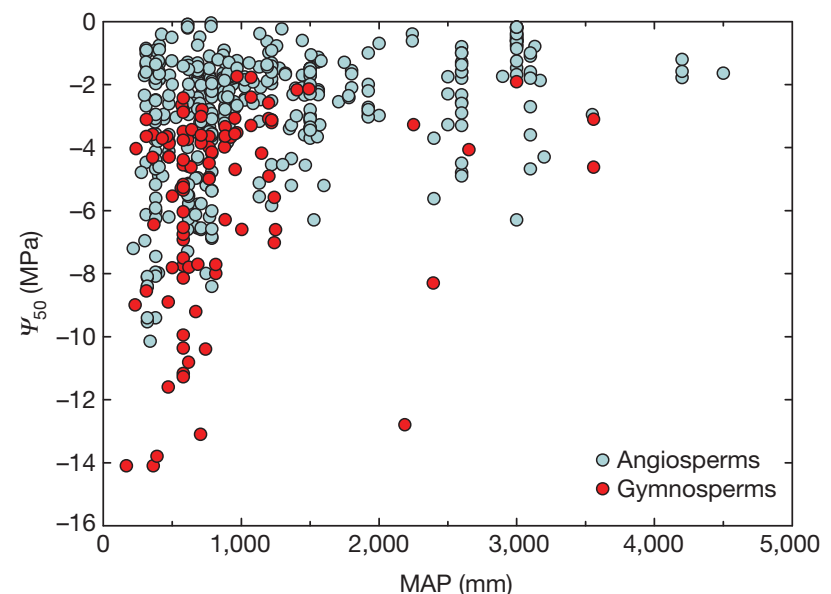

Figure 3 Embolism resistance as a function of mean annual precipitation for $\mathbf{3 8 4}$ angiosperm and $\mathbf{9 6}$ gymnosperm species. Each point represents one species. A generalized model indicated that embolism resistance $\left(\Psi_{50}\right)$ was significantly related $(P<0.00001)$ to mean annual precipitation $(\mathrm{MAP})$ for angiosperms and gymnosperms (see Methods for details), with decreasing resistance to embolism corresponding to increasing rainfall. The full data set is available in Supplementary Table 1.

It is clear from Fig. 3 that $\Psi_{50}$ and MAP are decoupled in certain cases, implying that some species growing in drier environments escape from water stress, therefore alleviating the need for high embolism resistance. There are many examples in the literature of species with low embolism resistance growing in areas of low rainfall or seasonal drought. This includes riparian and ground-water-dependent vegetation $^{10}$, and drought-deciduous trees in tropical dry forests ${ }^{27}$. These species avoid very negative $\Psi_{\mathrm{x}}$ by some combination of predictable access to ground water (deep roots), internal water storage and reduced leaf area or other shifts in biomass allocation ${ }^{10,23,28}$. Although these adjustments decouple $\Psi_{50}$ and $\Psi_{\text {min }}$ from MAP, it seems that the majority of species operate close to their functional limits. They are therefore exposed to xylem failure during anomalously low rainfall in a manner that is largely independent of rainfall region and biome (Fig. 2).

The convergence on a 'risky' hydraulic strategy exhibited by many species can be understood as the result of a trade-off that balances growth with protection against risk of mortality in a given environment ${ }^{17,21,25}$. Thus, a low safety margin to $\Psi_{50}$ also indicates that stomatal regulation takes full advantage of the range of xylem pressures that are within the tolerance of the hydraulic system of that species. This stomatal behaviour carries with it the benefit of increased carbon gain but may come at the cost of extensive loss of photosynthetic area or death.

A fundamental question concerns the plasticity and genetic diversity of embolism resistance within species ${ }^{22,29,30}$. If the tight link between embolism resistance and water availability is the product of natural selection over many generations and adaptation is limited by a long generation cycle of perennial plants, then the rapid pace of climate change may outstrip the capacity of populations to adapt. This could lead to long-term reductions in net primary productivity of forest systems, loss of biodiversity and changes to the composition of forest and woodland communities. Although it is evident that multiple mechanisms (hydraulic failure, carbohydrate depletion and insect attack) are involved in drought-induced mortality, these mechanisms are highly interdependent ${ }^{11,12}$. Embolism formation is a key mechanism of vegetation shifts and forest decline because it sets the thresholds for stomatal closure, leading to limitations on photosynthesis, increased heat and light damage, and a run-down of carbohydrate reserves over time. Our findings demonstrate the necessity of integrating long-term monitoring of $\Psi_{\min }$ with measurements of embolism resistance and safety margins. The inclusion of these data in process-based vegetation models will improve the accuracy with which the responses of forest ecosystems to climate change can be predicted.

\section{METHODS SUMMARY}

Xylem traits were compiled from published and unpublished sources (Supplementary Table 1). When values of $\Psi_{50}$ and $\Psi_{88}$ were not reported in numerical form, they were extracted from published graphs of vulnerability curves. $\Psi_{\min }$ data are the minimum midday water potential recorded for each species in the field, indicating a seasonal, rather than daily minimum value. Two types of safety margins were calculated: the $\Psi_{50}$ margin $\left(\Psi_{\min }-\Psi_{50}\right)$ and the $\Psi_{88} \operatorname{margin}\left(\Psi_{\min }-\Psi_{88}\right)$ (Supplementary Fig. 1).

Data in Fig. 1 were binned in 1.0-MPa increments of $\Psi_{50}$. Bins were pooled with the next lowest bin if they contained only one sample. Regression lines in Fig. 1 presented are for raw data to avoid bias associated with uneven bin size. Differences in biome means for safety margin were analysed using a general linear model, with differences between angiosperms and gymnosperms considered separately. Climate data were taken from the paper in which $\Psi_{50}$ data were published, or from the WorldClim database or CRU climate database, whichever gave an elevation closest to that given in the paper. Relationships between $\Psi_{50}$ and climate variables (MAP, aridity index, mean precipitation of the driest quarter) were analysed using a generalized model assessed by restricted maximum likelihood in which variance was simultaneously modelled as a power function of the same climate variables. Quantile regression was also used to assess the boundary relationship between $\Psi_{50}$ and climate variables. As $\Psi_{50}$ is a negative variable, the $10 \%$ quantile was used, which is equivalent to the $90 \%$ quantile for a positive response variable.

Forest biomes were assigned based on site descriptions contained in the primary sources. We defined 'forest' broadly to include Mediterranean, savanna and woodland environments that are not commonly classified as forests. The data set therefore encompasses tree, shrub and liana species from vegetation communities with a significant component of woody plants.

Full Methods and any associated references are available in the online version of the paper.

\section{Received 21 January; accepted 18 October 2012.}

Published online 21 November 2012.

1. Allen, C. D. et al. A global overview of drought and heat-induced tree mortality reveals emerging climate change risks for forests. For. Ecol. Manage. 259, 660-684 (2010).

2. Brodribb, T. J. \& Cochard, H. Hydraulic failure defines the recovery and point of death in water-stressed conifers. Plant Physiol. 149, 575-584 (2009).

3. Kursar, T. A. et al. Tolerance to low leaf water status of tropical tree seedlings is related to drought performance and distribution. Funct. Ecol. 23, 93-102 (2009).

4. McDowell, N. etal. Mechanisms of plant survival and mortality during drought: why do some plants survive while others succumb to drought? New Phytol. 178, 719-739 (2008).

5. Allison, I. et al. The Copenhagen Diagnosis: Updating the World on the Latest Climate Science (Elsevier, 2009).

6. Zhang, X. et al. Detection of human influence on twentieth-century precipitation trends. Nature 448, 461-465 (2007).

7. Meir, P. \& Woodward, F. I. Amazonian rain forests and drought: response and vulnerability. New Phytol. 187, 553-557 (2010).

8. Phillips, O. L. et al. Drought sensitivity of the amazon rainforest. Science $\mathbf{3 2 3}$, 1344-1347 (2009).

9. Engelbrecht, B. M. J. et al. Drought sensitivity shapes species distribution patterns in tropical forests. Nature 447, 80-82 (2007).

10. Pockman, W. T.\& Sperry, J.S. Vulnerability to xylem cavitation and the distribution of Sonoran desert vegetation. Am. J. Bot. 87, 1287-1299 (2000).

11. McDowell, N. G. et al. The interdependence of mechanisms underlying climatedriven vegetation mortality. Trends Ecol. Evol. 26, 523-532 (2011).

12. Anderegg, W. R. L. et al. The roles of hydraulic and carbon stress in a widespread climate-induced forest die-off. Proc. Natl Acad. Sci. USA 109, 233-237 (2012).

13. Breshears, D. D. et al. Regional vegetation die-off in response to global-changetype drought. Proc. Natl Acad. Sci. USA 102, 15144-15148 (2005).

14. Zhao, M. \& Running, S. W. Drought-induced reduction in global terrestrial net primary production from 2000 through 2009. Science 329, 940-943 (2010)

15. Lewis, S. L. Tropical forests and the changing earth system. Phil. Trans. R. Soc. $B$ 361, 195-210 (2006)

16. Pockman, W. T., Sperry, J. S. \& O'Leary, J. W. Sustained and significant negative water pressure in xylem. Nature 378, 715-716 (1995).

17. Tyree, M. T.\& Sperry, J. S. Vulnerability of xylem to cavitation and embolism. Annu. Rev. Plant Phys. Mol. Bio. 40, 19-38 (1989)

18. Sperry, J.S., Hacke, U. G. \& Pittermann, J. Size and function in conifer tracheids and angiosperm vessels. Am. J. Bot. 93, 1490-1500 (2006).

19. Maherali, H., Pockman, W. T. \& Jackson, R. B. Adaptive variation in the vulnerability of woody plants to xylem cavitation. Ecology 85, 2184-2199 (2004). 
20. Delzon, S., Douthe, C. Sala, A \& Cochard, H. Mechanism of water-stress induced cavitation in conifers: bordered pit structure and function support the hypothesis of seal capillary-seeding. Plant Cell Environ. 33, 2101-2111 (2010).

21. Sperry, J. S., Adler, F. R., Campbell, G. S. \& Comstock, J. P. Limitation of plant water use by rhizosphere and xylem conductance: results from a model. Plant Cell Environ. 21, 347-359 (1998).

22. Alder, N. N., Sperry, J. S. \& Pockman, W. T. Root and stem xylem embolism, stomatal conductance, and leaf turgor in Acer grandidentatum populations along a soil moisture gradient. Oecologia 105, 293-301 (1996).

23. Meinzer, F. C., Johnson, D. M., Lachenbruch, B., McCulloh, K. A. \& Woodruff, D. R. Xylem hydraulic safety margins in woody plants: coordination of stomatal control of xylem tension with hydraulic capacitance. Funct. Ecol. 23, 922-930 (2009).

24. Brodersen, C. R., McElrone, A. J., Choat, B., Matthews, M. A. \& Shackel, K. A. The dynamics of embolism repair in xylem: in vivo visualizations using high-resolution computed tomography. Plant Physiol. 154, 1088-1095 (2010).

25. Brodribb, T. J., Bowman, D. J. M. S., Nichols, S., Delzon, S. \& Burlett, R. Xylem function and growth rate interact to determine recovery rates after exposure to extreme water deficit. New Phytol. 188, 533-542 (2010).

26. Martínez-Vilalta, J., Sala, A. \& Piñol, J. The hydraulic architecture of Pinaceae-a review. Plant Ecol. 171, 3-13 (2004).

27. Choat, B., Ball, M. C., Luly, J. G. \& Holtum, J. A. M. Hydraulic architecture of deciduous and evergreen dry rainforest tree species from north-eastern Australia. Trees 19, 305-311 (2005).
28. Scholz, F. G., Phillips, N. G., Bucci, S. J., Meinzer, F. C. \& Goldstein, G. in Size- and Age-Related Changes in Tree Structure and Function Vol. 4 (eds Meinzer, F. C. et al) 341-361 (Springer, 2011).

29. Lamy, J. B. et al. Uniform selection as a primary force reducing population genetic differentiation of cavitation resistance across a species range. PLOS ONE 6, e23476 (2011).

30. Wortemann, R. et al. Genotypic variability and phenotypic plasticity of cavitation resistance in Fagus sylvatica L. across Europe. Tree Physiol. 31, 1175-1182 (2011).

Supplementary Information is available in the online version of the paper.

Acknowledgements We thank the ARC-NZ Vegetation Function Network for hosting the original working group from which the data set was compiled. We are grateful to the Alexander von Humboldt Foundation for supporting B.C. during preparation of the manuscript.

Author Contributions B.C. and S.J. led the initial working group and coordinated the analysis and write-up of the work. B.C., S.J., T.J.B., H.C., S.D., R.B., S.J.B., T.S.F., S.M.G., U.G.H., A.L.J., F.L., H.M., J.M.-V., S.M., M.M., P.J.M., A.N., J.P., R.B.P., J.S.S., M.W., I.J.W. and A.E.Z. contributed to compilation and organization of the data set and writing of the manuscript. S.M.G. and I.J.W. extracted climate data from the WorldClim and CRU climate databases. H.M., M.M. and J.M.-V. assisted in statistical analyses of the data set.

Author Information Reprints and permissions information is available at www.nature.com/reprints. The authors declare no competing financial interests. Readers are welcome to comment on the online version of the paper. Correspondence and requests for materials should be addressed to S.J. (steven.jansen@uni-ulm.de). 


\section{METHODS}

The resistance of a species to embolism is described by a vulnerability curve, which shows the percentage loss of hydraulic conductivity (PLC, \%) as a function of decreasing xylem pressure $\left(\Psi_{\mathrm{x}}, \mathrm{MPa}\right)$; that is, as plant drought stress increases (Supplementary Fig. 1). We define three parameters on this curve: first, the xylem pressure at which $50 \%$ of conductivity is lost $\left(\Psi_{50}, \mathrm{MPa}\right)$; second, the xylem pressure at which $88 \%$ of conductivity is lost $\left(\Psi_{88}, \mathrm{MPa}\right)$, which represents the upper inflection of the curve; and third, the slope of the curve between $\Psi_{50}$ and $\Psi_{88}$.

The most negative $\Psi_{\mathrm{x}}$ observed for a species is defined as $\Psi_{\text {min }}(\mathrm{MPa})$. 'Lethal' $\Psi_{\min }$ values reported in manipulative trials were not included in the database. If $\Psi_{\min }$ is equal to $\Psi_{50}$, this means that the plant has lost $50 \%$ of its hydraulic capacity. $\Psi_{50}$ is the most commonly used index of xylem embolism resistance in literature and represents the steepest part of the vulnerability curve, meaning that even a slight drop in $\Psi_{\mathrm{x}}$ will result in a substantial reduction in hydraulic function. However, $\Psi_{50}$ does not represent the mortality point per se because the $\Psi_{\mathrm{x}}$ value corresponding to lethal levels of embolism may vary among species, depending on the water-use strategy of the whole plant. In our data set, $42 \%$ of the angiosperm species (versus $6 \%$ of the gymnosperm species) show $\Psi_{\min }$ values below $\Psi_{50}$

A hydraulic safety margin was defined as the difference between $\Psi_{\min }$ and $\Psi_{50}$ (Supplementary Fig. 1 and Fig. 2a). An alternative, more conservative safety margin was defined as the difference between $\Psi_{\min }$ and $\Psi_{88}$ (Fig. 2b). Both the $\Psi_{50}$ and the $\Psi_{88}$ safety margins should be interpreted as relative vulnerability indices with a low (or even negative) value indicating a higher-risk strategy for hydraulic failure compared to a high safety margin.

The data set was compiled from published papers and unpublished results of the authors (see Supplementary Table 1), including 480 species from 172 sites, with 384 angiosperm and 96 gymnosperm species. Genera and species names were checked for orthography and synonymy using TaxonScrubber (version 2.1; www.salvias net/pages/taxonscrubber.html), the International Plant Names Index (www. ipni.org), Tropicos (www.tropicos.org), Phylomatic (www.phylodiversity.net/ phylomatic/) and the Angiosperm Phylogeny Website (http://www.mobot.org/ $\mathrm{MOBOT} / \mathrm{research} / \mathrm{APweb} /$ ). Samples based on measurements of roots, petioles or trunks were removed for the analyses. Data presented for $\Psi_{50}$ and $\Psi_{88}$ were from distal branches (approximately $0.5-1.2 \mathrm{~cm}$ in diameter) of 1 - to 3-year-old stems and therefore represent a conservative estimate of embolism resistance, as roots and leaves are normally less embolism resistant than stems within a species. All but $2 \%$ of the samples are from mature trees with $2 \%$ of samples coming from saplings or seedlings. PET data were extracted from the Global Aridity Index
(Global-Aridity) and the Global Potential Evapo-Transpiration (Global-PET) Geospatial Database (http://www.cgiar-csi.org/2010/04/134/).

The data set was then filtered for the target parameters. The data set was first filtered for samples in which both $\Psi_{50}$ and $\Psi_{\text {min }}$ were available, and filtered separately for $\Psi_{50}$ and MAP, which accounted for the majority of samples initially included. Only $\Psi_{88}$ data for $\Psi_{\text {min }}$ filtered species were included. Relationships between $\Psi_{50}$ and climate variables (MAP, aridity index, mean precipitation of the driest quarter) were analysed using a generalized model assessed by restricted maximum likelihood in which variance was simultaneously modelled as a power function of the same climate variables. Quantile regression was also used to assess the boundary relationship between $\Psi_{50}$ and climate variables. As $\Psi_{50}$ is a negative variable, the $10 \%$ quantile was used, which is equivalent to the $90 \%$ quantile for a positive response variable.

$\Psi_{50}$ and $\Psi_{\text {min }}$ were collected from the same population of plants with the exception of a few cases in which $\Psi_{\text {min }}$ data were extracted from another study or from unpublished sources. In $67 \%$ of our samples $\Psi_{\min }$ was measured as xylem or stem water potential, that is, the leaves were covered with plastic and aluminium foil such that leaf and stem water potentials were equilibrated. In the remaining 33\% of cases $\Psi_{\min }$ was measured as leaf water potential. In this case, $\Psi_{\mathrm{x}}$ may be less negative than leaf water potential because of the pressure drop across the leaf hydraulic pathway caused by transpiration. Because of the difference in leaf and xylem water potential it is possible the amount of embolism in the stem could be overestimated, that is, the safety margin would appear narrower than it actually was.

However, there are two main reasons why this issue would not alter the results. First, $\Psi_{\min }$ has most probably been underestimated in most cases because we lack continuous long-term data sets of water potential. Second, $48 \%$ of the species for which leaf water potential was recorded were below $-2.0 \mathrm{MPa}$. At this point the stomata are assumed to be closed in isohydric species (that is, species that close their stomata to prevent $\Psi_{\mathrm{x}}$ from dropping) and thus leaf water potential would be close to equilibrium with stem water potential. To estimate the magnitude of any potential error, we correlated $\Psi_{\text {min }}$ pre-dawn and $\Psi_{\min }$ midday for studies in which both were available. Although there is equilibrium for $\Psi_{\min }$ pre-dawn between leaves and stems, in theory there should be a more significant drop in $\Psi_{\min }$ midday for leaves than for stems. However, the slope of the relationship between $\Psi_{\text {min }}$ midday and $\Psi_{\min }$ pre-dawn was statistically indistinguishable for leaves and stems, indicating that no bias was introduced by combining leaf and stem $\Psi_{\text {min }}$ data (analysis of covariance (ANCOVA) interaction term, $F_{1,112}=0.944, P=0.333$ ). 